\title{
FREE IDEALS OF ONE-RELATOR GRADED LIE ALGEBRAS
}

\author{
JOHN P. LABUTE
}

\begin{abstract}
In this paper we show that a one-relator graded Lie algebra $\mathfrak{g}=$ $L /(r)$, over a principal ideal domain $K$, has a homogeneous ideal $\mathfrak{h}$ with $\mathfrak{g} / \mathfrak{h}$ a free $K$-module of finite rank if the relator $r$ is not a proper multiple of another element in the free Lie algebra $L$. As an application, we deduce that the center of a one-relator Lie algebra over $K$ is trivial if the rank of $L$ is greater than two. As another application, we find a new class of one-relator pro-p-groups which are of cohomological dimension 2 .
\end{abstract}

\section{STATEMENT OF RESULTS}

Let $K$ be a principal ideal domain and let $L$ be the free Lie algebra over $K$ on the set $X$. Let $r$ be a nonzero element of $L$ and let $\mathfrak{g}=L /(r)$, where $(r)$ is the ideal of $L$ generated by $r$. We also assume that a grading of $L$ is given in which the elements of $X$ are homogeneous of degree $\geq 1$. The main result of this paper is the following:

Theorem 1. If $r$ is homogeneous of degree $d$ and $r \notin \mathfrak{m} L$ for every maximal ideal $\mathfrak{m}$ of $K$, there exists a homogeneous ideal $\mathfrak{h}$ of $\mathfrak{g}=L /(r)$ and homogeneous elements $g_{1}, \ldots, g_{n} \in \mathfrak{g}$ of degree $<d$ such that $\mathfrak{h}$ is a free Lie algebra and $\mathfrak{g} / \mathfrak{h}$ a free $K$-module with basis the images of $g_{1}, \ldots, g_{n}$ in $\mathfrak{g} / \mathfrak{h}$. If $\operatorname{Card}(X)>2$, or $X=\left\{x_{1}, x_{2}\right\}$ and $r \neq a x_{1}+b x_{2}, c\left[x_{1}, x_{2}\right]$, the rank of $\mathfrak{h}$ is at least 2 .

Theorem 1 is proved by the elimination method, using new results on the matrix of the adjoint representation of a free Lie algebra with respect to certain weighted Hall bases. These results are valid over any commutative ring $K$ and yield Theorem 1 for certain types of relators even when $K$ is not a PID.

Theorem 1 is actually the result of an attempt to answer a question a posed to us by Hyman Bass. This question, which was prompted by joint work of Bass and Lubotsky [2], was the following: "Let $\mathfrak{g}$ be a Lie algebra (over a field $K$ ) with $d$ generators and one (homogeneous) relator. If $d>2$, is the center of $\mathfrak{g}$ trivial?" The analogous question for one-relator groups was answered in the affirative by Murasugi [6]. The following theorem answers this question in the affirmative when $K$ is a field or when $K$ is a PID and $r$ is homogeneous.

Received by the editors June 13,1993 and, in revised form, November 8, 1993 and December 13, 1993.

1991 Mathematics Subject Classification. Primary 17B01, 17B40, 17B65, 17B70; Secondary 20E18, 17B55.

Partially supported by Quebec FCAC Grant 88-EQ-2489. 
Whether they remain true when $K$ is a PID and $r$ is non-homogeneous is an open question (even for $K=\mathbb{Z}$ ).

Theorem 2. Suppose that either $K$ is a field or $K$ is a PID and $r$ is homogeneous. If the center of the Lie algebra $\mathfrak{g}=L /(r)$ is not trivial then $X=\left\{x_{1}, x_{2}\right\}$ and either $r=a x_{1}+b x_{2}$ or $r=a\left[x_{1}, x_{2}\right]$.

Corollary. If $K$ is a field and the center of $\mathfrak{g}=L /(r)$ is nontrivial then $\mathfrak{g}$ is isomorphic to $K$ or $K \times K$.

The following result was pointed out to us by Warren Dicks. We thank him for allowing us to reproduce his argument here.

Theorem 3. If $\mathfrak{g}=L /(r)$ is a one-relator Lie algebra over a field $K$, there exists a free subalgebra $\mathfrak{h}$ of $\mathfrak{g}$ of finite codimension.

To see this, let $r_{d}$ be the highest degree form of $r$ for the usual grading of $L$ and apply Theorem 1 to get a free Lie subalgebra $\mathfrak{h}^{\prime}$ of $\mathfrak{g}^{\prime}=L /\left(r_{d}\right)$ of finite codimension. Let $h_{0}$ be a lifting of $\mathfrak{h}^{\prime}$ to a free Lie subalgebra of $L$. By a result of Sirsov [8], the (graded) ideal of highest degree forms of elements of $(r)$ is generated by $r_{d}$ and so $\mathfrak{h}_{0} \cap(r)=0$. The image $\mathfrak{h}$ of $\mathfrak{h}_{0}$ in $g$ is therefore a free Lie subalgebra of $\mathfrak{g}$ which is also easily seen to be of finite codimension.

In the last section of this paper we extend the elimination method to pro$p$-groups. Using Theorem 1 , we find a new class of one-relator pro-p-groups which are of cohomological dimension 2.

\section{Weighted Hall Sets}

Let $X$ be a set and let $M(X)$ be the free magma on $X$. Every $u \in$ $M(X), u \notin X$ can be written uniquely as a product $\alpha(u) \beta(u)$ with $\alpha(u), \beta(u)$ $\in M(X)$. Let $\ell=\ell_{X}: M(X) \rightarrow \mathbb{N}$ be the unique mapping such that $\ell(x)=1$ for all $x \in X$ and $\ell(u v)=\ell(u)+\ell(v)$ for all $u, v \in M(X) ; \ell(u)$ is called the length of $u$. If $u_{1}, \ldots, u_{n} \in M(X)$ we define $p_{n}=u_{n} \cdots u_{2} u_{1}$ inductively by $p_{1}=u_{1}$ and $p_{i+1}=u_{i+1} p_{i}$ for $i>1$. If $u_{2}=\cdots=u_{n}=a$ and $u_{1}=b$, we denote $p_{n}$ by $a^{n-1} b$. Every $u \in M(X)$ can be uniquely written in the form $u=u_{1} u_{2} \cdots u_{n} x$ with $u_{i} \in M(X), x \in X$ and $n \geq 0$. We call this the canonical (right-normed) decompostion of $u$ in $M(X)$.

For each $x \in X$ let $n_{x} \in \mathbb{N}^{*}=\mathbb{N}-\{0\}$ and let $\omega$ be the unique mapping of $M(X)$ into $\mathbb{N}^{*}$ such that $\omega(x)=n_{x}$ if $x \in X$ and $\omega(u v)=\omega(u)+\omega(v)$ for $u, v \in M(X)$. We will call $\omega(u)$ the weight of $u$ with respect to the weight system $\left(n_{x}\right)_{x \in X}$ and $\omega$ the associated weight function. The weight system is completely determined by $\omega$ and we will ususally let $\omega$ denote it. If $X$ is ordered we say that the weight system is compatible with this ordering if $x<y \Longrightarrow \omega(x) \leq \omega(y)$.

A weighted Hall set, relative to a well-ordered set $X$ and compatible weight system $\omega$, is a subset $H$ of $M(X)$ together with a well-ordering < of $H$ such that

(1) $X$ is an ordered subset of $H$;

(2) For $u, v \in H, \omega(u)<\omega(v) \Longrightarrow u<v$;

(3) For all $u \in M(X)$ with $\ell(u)>1, u \in H \Longleftrightarrow \alpha(u), \beta(u) \in H$ and $\alpha(u)<\beta(u)$ with $\alpha(u)>\alpha(\beta(u))$ if $\ell(\beta(u))>1$.

(4) If $u, v \in H$ with $\omega(u)=\omega(v)$ and $\ell(u), \ell(v)>1$ then $u<v$ iff $\beta(u)<\beta(v)$ or $\beta(u)=\beta(v)$ and $\alpha(u)<\alpha(v)$. 
If $\omega(x)=1$ for all $x$, we have $\ell=\omega$ and we recover the usual definition of a Hall set. A weighted Hall set $H$ is said to be of finite type if for any $k \in \mathbb{N}^{*}$ there are only finitely many $u \in H$ of weight $k$. This is equivalent to requiring that, for any $k,\{x \in X \mid \omega(x)=k\}$ be a finite set; a weight function with this property is also said to be of finite type. If $u \in M(X)$ with canonical decomposition $u_{1} u_{2} \cdots u_{n} x$, we have $u \in H$ iff $u_{1}, \cdots, u_{n} \in H$, $u_{1} \geq u_{2} \geq \cdots \geq u_{n}<x$ and $u_{i-1}<u_{i} \cdots u_{n} x$ for $2 \leq i \leq n$. If $u \in H$, we will call its canonical decomposition in $M(X)$ the canonical decomposition of $u$ in $H$.

Proposition 1. Given any well-ordered set $X$ and a compatible weight system $\omega$ on $X$, there exists a weighted Hall set $H$ relative to $X, \omega$.

Proof. We construct, by induction on $n$, well-ordered subsets $W_{n}$ of $M(X)$ consisting of elements of weight $n$ :

(a) We let $W_{1}$ consist of those elements of $X$ which are of weight 1 .

(b) Let $n \geq 2$ so that the well-ordered sets $W_{1}, \ldots, W_{n-1}$ are already constructed. Let $W_{n-1}^{\prime}=W_{1} \cup \cdots \cup W_{n-1}$ together with the well-ordering which induces the given orderings on $W_{1}, \ldots, W_{n-1}$ and such that $u<v$ if $\omega(u)<$ $\omega(v)$. Let $W_{n}$ consist of those elements of $X$ of weight $n$ together with the products $a b$ of weight $n$ with $a, b \in W_{n-1}^{\prime}, a<b$ and $a>\alpha(b)$ if $\ell(b)>1$. If $a b, a^{\prime} b^{\prime} \in W_{n}, x \in X \cap W_{n}$, let $a b<x$ and let $a b<a^{\prime} b^{\prime}$ if $b<b^{\prime}$ or if $b=b^{\prime}$ and $a<a^{\prime}$. This defines a well-ordering of $W_{n}$.

The required well-ordered set $H$ is the union of the $W_{i}$.

Proposition 2. Suppose that $u, v, u v \in H$. If $u_{1}, u_{1} v \in H$ and $u<u_{1}$ (resp. $v_{1}, u v_{1} \in H$ and $\left.v<v_{1}\right)$ then $u v<u_{1} v$ (resp. $\left.u v<u v_{1}\right)$.

Proof. Simply note that $u<u_{1}$ implies that $\omega(u) \leq \omega\left(u_{1}\right)$. If the inequality is strict, we have $\omega(u v)<\omega\left(u_{1} v\right)$, and so $u v<u_{1} v$. Otherwise, $u v$ and $u_{1} v$ have the same weight and $u<u_{1}$, which implies $u v<u_{1} v$.

Now let $H$ be any weighted Hall set relative to the set $X$ and a given weight system $\omega$. Let $x_{1}$ be the smallest element of $X$; this element is also the smallest of $H$. Let $H_{1}=H-\left\{x_{1}\right\}$ and let $X_{1}$ be the subset of $H_{1}$ consisting of those elements which cannot be written as a product of 2 elements of $H_{1}$. We have

$$
X_{1}=\left\{x_{1}^{j} x \mid x \in X, x \neq x_{1}, j \geq 0\right\} .
$$

Identify $M\left(X_{1}\right)$ with its canonical image in $M(X)$ and let $\omega_{1}$ be the restriction of $\omega$ to $M\left(X_{1}\right)$. Let $\ell_{1}$ be the length function on $M\left(X_{1}\right)$.

Proposition 3. The set $H_{1}$, with the induced ordering from $H$, is a weighted Hall set relative to $X_{1}, \omega_{1}$. If $H$ is of finite type then so is $H_{1}$.

Proof. We need only prove the first assertion. Since (1), (2) and (4) follow immediately, it suffices to verify condition (3).

Let $u \in M\left(X_{1}\right)$ with $\ell_{1}(u)>1$. Then $u=u_{1} u_{2}$ with $u_{1}, u_{2} \in M\left(X_{1}\right)$. If $u \in H_{1}$ then $u_{1}, u_{2} \in H$ with $u_{1}<u_{2}$ since $H_{1} \subset H$. Since $x_{1} \notin M\left(X_{1}\right)$ we obtain $u_{1}, u_{2} \in H_{1}$. If $u_{2}=u_{2}^{\prime} u_{2}^{\prime \prime}$ with $u_{2}^{\prime}, u_{2}^{\prime \prime} \in H_{1}$, we have $u_{1}>u_{2}^{\prime}$ since $H_{1} \subset H$.

Conversely, suppose $u=u_{1} u_{2}$ with $u_{1}, u_{2} \in H_{1}, u_{1}<u_{2}$. If $u_{2}=u_{2}^{\prime} u_{2}^{\prime \prime}$ with $u_{2}^{\prime}, u_{2}^{\prime \prime} \in H_{1}$ then $u_{1}>u_{2}^{\prime}$ implies that $u \in H$ and so $u \in H_{1}$ since 
$u \neq x_{1}$. If $\ell_{1}\left(u_{2}\right)=1$ and $\ell\left(u_{2}\right)>1$, we have $u_{2}=x_{1} v$ with $v \in H_{1}$. Since $u_{1} \geq x_{1}$ we have $u \in H$ and so $u \in H_{1}$.

Let $u$ be any element of $H_{1}$ and let $u_{1} u_{2} \cdots u_{k} y$ be its canonical decomposition in $H_{1}$. Let $\gamma(u) \in H_{1}$ be the element $u_{1} u_{2} \cdots u_{k} y^{\prime}$ with $y^{\prime}=x_{1} y$; this product is the canonical decomposition of $\gamma(u)$ in $H_{1}$. We will call $\gamma$ the dominance function for $H$. We say that $H$ has the dominance property if, for all $x \in X$ and $u \in H$ with $\omega(x)=\omega(u)>1$ and $x<u$, we have $x<\gamma(\beta(u))$ if $\ell(u)>1$. If $\omega=\ell$, then $H$ has the dominance property since this condition holds vacuously. For the same reason, the weighted Hall set constructed in Proposition 1 has the dominance property.

Proposition 4. The Hall set $H$ has the dominance property iff, for all $u, v \in H_{1}$,

$$
u<v \Longrightarrow \gamma(u)<\gamma(v) \text {. }
$$

Proof. ( $\Longrightarrow$ ) Since the assertion is trivial if $u, v \in X_{1}$ or if $\omega(u)<\omega(v)$, we may assume that not both $u, v$ lie in $X_{1}$ and that $u, v$ have the same weight. We proceed by induction on $\omega(u)$.

If $u=u_{1} u_{2}, v=v_{1} v_{2}$ in $H_{1}$ then $\gamma(u)=u_{1} \gamma\left(u_{2}\right), \gamma(v)=v_{1} \gamma\left(v_{2}\right)$. If $u_{2}=v_{2}$, we are done. If $u_{2}<v_{2}$ then by induction on the degree we have $\gamma\left(u_{2}\right)<\gamma\left(v_{2}\right)$ which gives the required result.

Now suppose $u=u_{1} u_{2}$ in $H_{1}$ and $v \in X_{1}$. If $v \notin X$ then $v=x_{1} w$ for some $w \in X_{1}$. Since $u_{2}<w$ we have $\gamma\left(u_{2}\right)<\gamma(w)=v$ by induction on the degree. But then

$$
\gamma(u)=u_{1} \gamma\left(u_{2}\right)<x_{1} v=\gamma(v) .
$$

We may therefore assume $v \in X$. Since

$$
\omega(v)=\omega\left(u_{1}\right)+\omega\left(u_{2}\right) \geq \omega\left(x_{1}\right)+\omega\left(u_{2}\right)=\omega\left(\gamma\left(u_{2}\right)\right),
$$

we have $v \geq \gamma\left(u_{2}\right)$. But $v \neq \gamma\left(u_{2}\right)$ as $v \in X$ and we again obtain the desired result.

Finally, suppose $u \in X_{1}, v=v_{1} v_{2}$ in $H_{1}$. Since the case $u \notin X$ can be treated as above, we may assume that $u \in X$. Since $H$ has the dominance property, we have $u<\gamma\left(v_{2}\right)$ and so $\gamma(u)=x_{1} u<v_{1} \gamma\left(v_{2}\right)=\gamma(v)$.

( $\Longleftarrow$ ) Suppose $x \in X, u \in H$ with $x<u, \ell(u)>1$ and $\omega(x)=\omega(u)$. Let $u=a b$. Then $x_{1} x<a \gamma(b)$ which implies $x<\gamma(b)$ since $x \neq \gamma(b)$.

Proposition 5. If $H$ has the dominance property then so has $H_{1}$.

Proof. Let $\gamma_{1}$ be the dominance function for $H_{1}$, let $x_{2}$ be the smallest element of $X_{1}$, let $H_{2}=H_{1}-\left\{x_{2}\right\}$ and let $X_{2}$ be the indecomposable elements of $H_{1}$. Let $u \in X_{1}, v=v_{1} v_{2}$ in $H_{1}$ with $u<v$ and $\omega(u)=\omega(v)$. Then $u \in H_{2}$ and

$$
x_{1} u=\gamma(u)<\gamma(v)=v_{1} \gamma\left(v_{2}\right),
$$

which yields $u \leq \gamma\left(v_{2}\right)$. If we could show that $\gamma\left(v_{2}\right)<\gamma_{1}\left(v_{2}\right)$ then we would have

$$
\gamma_{1}(u)=x_{2} u<v_{1} \gamma_{1}\left(v_{2}\right)=\gamma_{1}(v) .
$$

Hence we are reduced to showing that $\gamma\left(v_{2}\right)<\gamma_{1}\left(v_{2}\right)$. In $H_{2}$ we have the canonical decomposition

$$
v_{2}=w_{1} w_{2} \cdots w_{t} z
$$


with $z=x_{2}^{i} x_{1}^{j} x, x \in X, x \neq x_{1}$ and $j \neq 0$ if $x=x_{2}$. Since

$$
\begin{aligned}
\gamma\left(v_{2}\right) & =w_{1} w_{2} \cdots w_{t} x_{2}^{i} x_{1}^{j+1} x, \\
\gamma_{1}\left(v_{2}\right) & =w_{1} w_{2} \cdots w_{t} x_{2}^{i+1} x_{1}^{j} x,
\end{aligned}
$$

we are reduced to showing that

$$
x_{2}^{i} x_{1}^{j+1} x<x_{2}^{i+1} x_{1}^{j} x .
$$

This is true if $\omega\left(x_{1}\right)<\omega\left(x_{2}\right)$ since then the right-hand side has a larger weight than the left. If $x_{1}, x_{2}$ have the same weight, we are reduced to showing that

$$
x_{1} x_{1}^{j} x<x_{2} x_{1}^{j} x .
$$

But this is true since $x_{1}<x_{2}$.

Corollary. If $H$ has the dominance property, is of finite type and $u \in H$, the set $\{v \in H \mid v \geq u\}$ is a weighted Hall set having the dominance property.

Let $L(X)$ be the free Lie algebra on $X$ over a commutative ring $K$. We have a canonical mapping $\psi$ of $M(X)$ into $L(X)$ such that $\psi(u v)=[\psi(u), \psi(v)]$. Let $H$ be any weighted Hall set relative to $X, \omega$.

Proposition 6. The mapping $\psi$ is injective on $H$ and the image of $H$ is a basis of $L(X)$ as a $K$-module.

Proof. Without loss of generality we may assume that $X$ is finite. Let $x_{1}$ be the smallest element of $X$. By the elimination theorem (cf. [1, §2.9, Proposition 10]) we have $L(X)=K x_{1} \oplus L\left(X_{1}\right)$. Repeating this argument a finite number of times, we obtain the result.

We identify $H$ with its image in $L(X)$ and let ad be the ajoint representation of $L(X)$; we have $\operatorname{ad}(u)(v)=[u, v]$.

Theorem 4. Suppose that $H$ has the dominance property and $u \in H_{1}$. Then

$$
\operatorname{ad}\left(x_{1}\right)(u)=\gamma(u)+w,
$$

where $w$ a linear combination of elements of $H_{1}$ which are smaller than $\gamma(u)$. Proof. Since the assertion is trivially true if $\ell_{1}(u)=1$ we may assume that $\ell_{1}(u)>1$. If $\ell_{1}(u)=2$ then $u=\left[u_{1}, u_{2}\right]$ with $u_{1}, u_{2} \in X_{1}, u_{1}<u_{2}$, and we have $\operatorname{ad}\left(x_{1}\right)(u)=\left[\gamma\left(u_{1}\right), u_{2}\right]+\left[u_{1}, \gamma\left(u_{2}\right)\right]$. If $\gamma\left(u_{1}\right)<u_{2}$ then $w=$ $\left[\gamma\left(u_{1}\right), u_{2}\right] \in H_{1}$ and $w<\left[u_{1}, \gamma\left(u_{2}\right)\right]=\gamma(u)$ since $u_{2}<\gamma\left(u_{2}\right)$. If $\gamma\left(u_{1}\right)>u_{2}$ then $\operatorname{ad}\left(x_{1}\right)(u)=-\left[u_{2}, \gamma\left(u_{1}\right)\right]+\gamma(u)$ and $w=\left[u_{2}, \gamma\left(u_{1}\right)\right] \in H_{1}, w<\gamma(u)$ since $u_{1}<u_{2}$ implies that $\gamma\left(u_{1}\right)<\gamma\left(u_{2}\right)$.

Assume that, if $v \in H_{1}$ with $2 \leq \ell_{1}(v) \leq k$, we have shown that $\operatorname{ad}\left(x_{1}\right)(v)=$ $\gamma(v)+w$, where $w \in H_{1}$ is a linear combination of terms $w^{\prime} \in H_{1}$ with $\ell_{1}\left(w^{\prime}\right)>1$ and

(1) $w^{\prime}<\gamma(v)$;

(2) $\alpha(v) \leq \alpha\left(w^{\prime}\right)<\beta\left(w^{\prime}\right)<v$;

(3) $\alpha(v) \geq \alpha\left(\alpha\left(w^{\prime}\right)\right)$ (resp. $\left.\alpha\left(\beta\left(w^{\prime}\right)\right)\right)$ if $\ell_{1}\left(\alpha\left(w^{\prime}\right)\right)>1$ (resp. $\ell_{1}\left(\beta\left(w^{\prime}\right)\right)>$ 1). 
This holds if $k=2$. We want to show that this then holds for $v=u$ with $\ell_{1}(u)=k+1$. We divide the proof into three cases.

Case 1: $u=[x, v]$ in $H_{1}$ with $x \in X_{1}, \ell_{1}(v)>1$. Then $x<v=[a, b]$, $a, b \in H_{1}, x \geq a$, and by induction $\operatorname{ad}\left(x_{1}\right)(v)=\gamma(v)+w$, where $w \in H_{1}$ is a linear combination of terms $w^{\prime}=\left[a^{\prime}, b^{\prime}\right] \in H_{1}$ with $\ell_{1}\left(w^{\prime}\right)>1$ satisfying (1), (2), (3). But then

$$
\begin{aligned}
\operatorname{ad}\left(x_{1}\right)(u) & =[\gamma(x), v]+[x, \gamma(v)]+w_{1} \\
& =\gamma(u)+[\gamma(x), v]+w_{1},
\end{aligned}
$$

where $w_{1}$ is a linear combination of terms $\left[x,\left[a^{\prime}, b^{\prime}\right]\right]$ with $\left[a^{\prime}, b^{\prime}\right]$ as above. We have to show that $[\gamma(x), v],\left[x,\left[a^{\prime}, b^{\prime}\right]\right]$ are linear combinations of terms $w^{\prime} \in H_{1}$ with $\ell_{1}\left(w^{\prime}\right)>1$ and satisfying (1), (2), (3) with $v$ replaced by $u$

Consider first the term $[\gamma(x), v]$. If $\gamma(x)<v$ then $[\gamma(x), v] \in H_{1}$ since $\gamma(x)>x \geq a$ and $[\gamma(x), v]<\gamma(u)=[x, \gamma(v)]$ since $v<\gamma(v)$. Since $x>a=$ $\alpha(v)$, condition (3) is satisfied, and (2) follows from $x<\gamma(x),[x, v]>v$. If $\gamma(x)>v$ then $[\gamma(x), v]=-[v, \gamma(x)]$ with $[v, \gamma(x)] \in H_{1}$ and $[v, \gamma(x)]<$ $\gamma(u)$ since $\gamma(x)<\gamma(v)$. Again condition (3) is satisfied since $x>a$. To prove (2) we note first that $\omega([x, v]) \geq \omega(\gamma(x))$ since $\gamma(x)=\left[x_{1}, x\right]$ and $x_{1}<x<v$. If $\omega([x, v])>\omega(\gamma(x))$, we have $[x, v]>\gamma(x)$, and if the weights are equal we have $[x, v]>\gamma(x)=\left[x_{1}, x\right]$ since $v>x$.

Now consider the terms $\left[x,\left[a^{\prime}, b^{\prime}\right]\right]$. If $x \geq a^{\prime}$, we have $\left[x,\left[a^{\prime}, b^{\prime}\right]\right] \in H_{1}$ and $\left[x,\left[a^{\prime}, b^{\prime}\right]\right]<[x, \gamma(v)] \gamma(u)=$ since $\left[a^{\prime}, b^{\prime}\right]<\gamma(v)$. Since $\omega([x, v]) \geq$ $\omega\left(\left[a^{\prime}, b^{\prime}\right]\right)$, we may assume in proving $(2)$ that $[x, v]$ and $\left[a^{\prime}, b^{\prime}\right]$ have equal weight. But then $[x, v]>\left[a^{\prime}, b^{\prime}\right]$ since $v>b^{\prime}$; this gives (2) and condition (3) follows from $x \geq a^{\prime}$. Now suppose that $x<a^{\prime}$. Then $\left[x, a^{\prime}\right],\left[x, b^{\prime}\right] \in H_{1}$ by (3) since $x \geq a$ and we have

$$
\left[x,\left[a^{\prime}, b^{\prime}\right]\right]=\left[\left[x, a^{\prime}\right], b^{\prime}\right]+\left[a^{\prime},\left[x, b^{\prime}\right]\right] .
$$

If $\left[x, a^{\prime}\right]<b^{\prime}$ then $\left[\left[x, a^{\prime}\right], b^{\prime}\right] \in H_{1}$ since $\left[x, a^{\prime}\right]>x \geq a$ and $a \geq \alpha\left(b^{\prime}\right)$ if $\ell_{1}\left(b^{\prime}\right)>1$. Now (1) holds since $b^{\prime}<\gamma(v)$ implies that $\left[\left[x, a^{\prime}\right], b^{\prime}\right]<$ $[x, \gamma(v)]=\gamma(u)$ and (3) holds since $x \geq a$ and $a \geq \alpha\left(b^{\prime}\right)$ if $\ell_{1}\left(b^{\prime}\right)>1$. To prove (2), we have to show $[x, v]>b^{\prime}$; but this is trivially true since $v>b^{\prime}$. If $\left[x, a^{\prime}\right]>b^{\prime}$ then $\left[b^{\prime},\left[x, a^{\prime}\right]\right] \in H_{1}$ and (3) follows as above. To prove (1), we have to show that $\left[b^{\prime},\left[x, a^{\prime}\right]\right]<[x,[a, \gamma(b)]]$. Since $b^{\prime}>x$, we have $\omega\left(b^{\prime}\right) \geq \omega(x)$ and so $\omega\left(\left[x, a^{\prime}\right]\right) \leq \omega([a, \gamma(b)])$. If we have strict inequality, we are done; so we may assume equality. But $b^{\prime}<\gamma(b)$ since $\left[a^{\prime}, b^{\prime}\right]<\gamma(v)=[a, \gamma(b)]$ and $a \leq a^{\prime}$. So $a^{\prime}<b^{\prime}<\gamma(b)$ which implies $\left[x, a^{\prime}\right]<\left[a^{\prime}, \gamma(b)\right]$ and we are done. Finally, $\left[a^{\prime},\left[x, b^{\prime}\right]\right] \in H_{1}$ and the same arguments as above yield (1),(2),(3).

Case 2: $u=[v, x]$ in $H_{1}$ with $x \in X_{1}, \ell_{1}(v)>1$. Then $v=[a, b]$ in $H_{1}$ and $\operatorname{ad}\left(x_{1}\right)(u)=\gamma(u)+[\gamma(v), x]+w_{1}$, where $w_{1}$ is a linear combination of terms $\left[w^{\prime}, x\right]$ with $w^{\prime}=\left[a^{\prime}, b^{\prime}\right]$ satisfying the same conditions as in Case 1 . We have to show that $\left[\left[a^{\prime}, b^{\prime}\right], x\right],[\gamma(v), x]$ are linear combinations of terms $w^{\prime} \in H_{1}$ with $\ell_{1}\left(w^{\prime}\right)>1$ and satisfying (1), (2), (3) with $v=u$.

Consider first the term $[\gamma(v), x]$. If $\gamma(v)<x$ then $[\gamma(v), x] \in H_{1}$ and is smaller than $\gamma(u)=[v, \gamma(x)]$ since $x<\gamma(x)$. Condition (2) holds since $[v, x]>x$, and (3) holds since $v>a$. If $\gamma(v)>x$ then $[\gamma(v), x]=$ $-[x, \gamma(v)]=-\left[x,[a, \gamma(b)]\right.$ and $\left[x,[a, \gamma(b)] \in H_{1}\right.$ since $x>a$. Moreover, $[x, \gamma(v)]<[v, \gamma(x)]=\gamma(u)$ since $v<x$ implies $\gamma(v)<\gamma(x)$, and $v>a$ gives 
(3). To prove (2) we have to show $[v, x]>\gamma(v)$. But $\omega([v, x]) \geq \omega(\gamma(v))$ and equality would imply $\omega(x)=\omega\left(x_{1}\right)$ which is impossible since $x>v$ and $\omega(v)>\omega(a) \geq \omega\left(x_{1}\right)$.

Now consider the terms $\left[\left[a^{\prime}, b^{\prime}\right], x\right]$. If $\left[a^{\prime}, b^{\prime}\right]<x$, we have $\left[\left[a^{\prime}, b^{\prime}\right], x\right] \in$ $H_{1}$ and $\left[\left[a^{\prime}, b^{\prime}\right], x\right]<[v, \gamma(x)]=\gamma(u)$, which gives (1), and (2) follows from $[v, x]>x$. Since $v>b^{\prime}>a^{\prime}$ we have (3). If $\left[a^{\prime}, b^{\prime}\right]>x$, we have $\left[\left[a^{\prime}, b^{\prime}\right], x\right]=-\left[x,\left[a^{\prime}, b^{\prime}\right]\right]$. Since $x>v>b^{\prime}>a^{\prime}$, we have $\left[x,\left[a^{\prime}, b^{\prime}\right]\right] \in$ $H_{1}$, and $\left[x, v^{\prime}\right]<[v, \gamma(x)]=\gamma(u)$ since $\left[a^{\prime}, b^{\prime}\right]<\gamma(v)<\gamma(x)$. This gives (1) and (3). Condition (2) follows from $\omega([v, x])>\omega\left(\left[a^{\prime}, b^{\prime}\right]\right)$.

Case 3: $u=\left[u_{1}, u_{2}\right]$ in $H_{2}$ with $\ell_{1}\left(u_{1}\right), \ell_{1}\left(u_{2}\right)>1$. Then $u_{1}=[a, b]$, $u_{2}=[c, d]$ in $H_{1}$ and

$$
\operatorname{ad}\left(x_{1}\right)(u)=\gamma(u)+\left[\gamma\left(u_{1}\right), u_{2}\right]+w_{1}+w_{2},
$$

where $w_{1}$ (resp. $w_{2}$ ) is a linear combination of terms $\left[\left[a^{\prime}, b^{\prime}\right], u_{2}\right] \in H_{1}$ (resp. $\left.\left[u_{1},\left[c^{\prime}, d^{\prime}\right]\right] \in H_{1}\right)$ with $w^{\prime}=\left[a^{\prime}, b^{\prime}\right] \in H_{1}$, (resp. $\left.w^{\prime}=\left[c^{\prime}, d^{\prime}\right] \in H_{1}\right)$ satisfying $\ell_{1}\left(w^{\prime}\right)>1$ and (1), (2), (3) with $v=u_{1}$ (resp. $v=u_{2}$ ). We have to show that the terms $\left[\gamma\left(u_{1}\right), u_{2}\right],\left[\left[a^{\prime}, b^{\prime}\right], u_{2}\right],\left[u_{1},\left[a^{\prime}, b^{\prime}\right]\right]$ are linear cominations of terms $w^{\prime} \in H_{1}$ with $\ell_{1}\left(w^{\prime}\right)>1$ and satisfying (1), (2), (3) with $v=u$.

Consider first the term $w=\left[\gamma\left(u_{1}\right), u_{2}\right]$. If $\gamma\left(u_{1}\right)<u_{2}$ then $w \in H_{1}$ as $\gamma\left(u_{1}\right)>u_{1}>c$. Since $w=[[a, \gamma(b)],[c, d]]$ and $u_{1}>a, c$ condition (3) holds. Also $w<\left[u_{1}, \gamma\left(u_{2}\right)\right]=\gamma(u)$, which gives $(1)$, and $\left[u_{1}, u_{2}\right]>u_{2}$ gives (2). If $\gamma\left(u_{1}\right)>u_{2}$ then $w=-\left[u_{2},[a, \gamma(b)]\right]$. Now $u_{2}>a$ since $\omega\left(u_{2}\right)>\omega(a)$ and so $z=\left[u_{2},[a, \gamma(b)]\right] \in H_{1}$. Now $z<\gamma(u)$ since $z=\left[u_{2}, \gamma\left(u_{1}\right)\right]$ and $\gamma\left(u_{1}\right)<\gamma\left(u_{2}\right)$. Finally, $u_{1} \geq a, c$ yields $(3)$, and $\omega\left(\left[u_{1}, u_{2}\right]\right)>\omega([a, \gamma(b)])$, $u_{1}<u_{2}$, gives $(2)$.

Now consider the term $w_{1}^{\prime}=\left[\left[a^{\prime}, b^{\prime}\right], u_{2}\right]$. If $\left[a^{\prime}, b^{\prime}\right]<u_{2}$ then $w_{1}^{\prime} \in H_{1}$ since $\left[a^{\prime}, b^{\prime}\right]>u_{1} \geq c$. Since $u_{2}<\gamma\left(u_{2}\right), w_{1}^{\prime}<\gamma(u)=\left[u_{1}, \gamma\left(u_{2}\right)\right]$, which gives (1). We have (2) since $u_{1}<\left[a^{\prime}, b^{\prime}\right],\left[u_{1}, u_{2}\right]>u_{2}$, and (3) holds since $u_{1}>a^{\prime}, c$. If $\left[a^{\prime}, b^{\prime}\right]>u_{2}$ then $w_{2}^{\prime}=-\left[u_{2},\left[a^{\prime}, b^{\prime}\right]\right]$ and $\left[u_{2},\left[a^{\prime}, b^{\prime}\right]\right] \in H_{1}$ since $u_{2}>u_{1}>a^{\prime}$. Since $\left[a^{\prime}, b^{\prime}\right]<\gamma\left(u_{1}\right)<\gamma\left(u_{2}\right)$, we have (1). Condition (2) holds since $\omega\left(\left[u_{1}, u_{2}\right]\right)>\omega\left(\left[a^{\prime}, b^{\prime}\right], u_{1}<u_{2}\right.$, and (3) holds as above.

Finally, consider the term $w_{2}^{\prime}=\left[u_{1},\left[c^{\prime}, d^{\prime}\right]\right]$. If $u_{1} \geq c^{\prime}$, we have $w_{2}^{\prime} \in H_{1}$, and (1) holds since $\left[c^{\prime}, d^{\prime}\right]<\gamma\left(u_{2}\right)$. Condition (2) holds since $\omega\left(\left[u_{1}, u_{2}\right]\right)>$ $\omega\left(\left[c^{\prime}, d^{\prime}\right]\right)$, and (3) holds since $u_{1} \geq a, c^{\prime}$. If $u_{1}<c^{\prime}$ we have

$$
w_{2}^{\prime}=\left[\left[u_{1}, c^{\prime}\right], d^{\prime}\right]+\left[c^{\prime},\left[u_{1}, d^{\prime}\right]\right.
$$

with $\left[u_{1}, c^{\prime}\right],\left[u_{1}, d^{\prime}\right] \in H_{1}$ since $u_{1} \geq c$ and $c \geq \alpha\left(c^{\prime}\right)$ (resp. $\left.\alpha\left(d^{\prime}\right)\right)$ if $\ell_{1}\left(c^{\prime}\right)>1$ (resp. $\ell_{2}\left(d^{\prime}\right)>1$ ). We have $\left[c^{\prime},\left[u_{1}, d^{\prime}\right] \in H_{1}\right.$, and (1) holds since $u_{1}<c^{\prime}$ implies that $\left[u_{1}, d^{\prime}\right]<\left[c^{\prime}, d^{\prime}\right]<\gamma\left(u_{2}\right)$. Condition (3) holds since $u_{1} \geq c$, and (2) holds since $u_{2}>d^{\prime}$ implies that $\left[u_{1}, u_{2}\right]>\left[u_{1}, d^{\prime}\right]$. Now consider the term $w=\left[\left[u_{1}, c^{\prime}\right], d^{\prime}\right]$. If $\left[u_{1}, c^{\prime}\right]<d^{\prime}$ then $w \in H_{1}$, and (1) holds since $d^{\prime}<\gamma\left(u_{2}\right)$. Condition (2) holds since $u>u_{2}>d^{\prime}$ and (3) follows from $u_{1} \geq c$. If $\left[u_{1}, c^{\prime}\right]>d^{\prime}$ then $z=-w=\left[d^{\prime},\left[u_{1}, c^{\prime}\right]\right] \in H_{1}$, and (1) follows from the above as $\left[u_{1}, c^{\prime}\right]<\left[u_{1}, d^{\prime}\right]$. We have (2) since $u_{1}<c^{\prime}<d^{\prime}$, $\left[u_{1}, c^{\prime}\right]<\left[u_{1}, d^{\prime}\right]<\left[u_{1}, u_{2}\right]=u$; we use that $d^{\prime}<u_{2}$. Condition (3) follows from $u_{1} \geq c$. 


\section{Proof OF THEOREM 1}

Choose an ordering of $X$ which is compatible with the given weight system $\omega$ on $X$ and let $H$ be a weighted Hall set with respect to $X, \omega$ which has the dominance property. We can write $r=c s+t$, where $c \in K-\{0\}, s \in H$ and $t$ is a linear combination of elements of $H$ which are strictly less than $s$. Assume that $c$ is a unit of $K$; this is true in the case that $K$ is a field. At this point we should like to point out that, under this assumption our proof is valid over any commutative ring $K$. Without loss of generality, we can assume $c=1$. If $r$ is a linear combination of elements of $X$ then $\mathfrak{g}$ is free; so we may assume that $r$ is not linear form. If $X=\left\{x_{1}, x_{2}\right\}$ and $r=\left[x_{1}, x_{2}\right]$ then again the theorem holds trivially. We may therefore assume that $r$ is not linear and that either $\operatorname{Card}(X)>2$ or $X=\left\{x_{1}, x_{2}\right\}$ and $r \neq c\left[x_{1}, x_{2}\right]$. We may also assume, without loss of generality, that $X$ is a finite set. Let $h_{1}<h_{2}<\ldots$ be the elements of $H$ and, for $i \geq 1$, let $H_{i}=\left\{h \in H_{i-1} \mid h>h_{i}\right\}$, where $H_{0}=H$. Let $X_{i}$ be the set of indecomposable elements of $H_{i}$; for $i \geq 1$ we have (setting $X_{0}=X$ )

$$
X_{i}=\left\{\operatorname{ad}\left(h_{i}\right)^{j}(x) \mid j \geq 0, x \in X_{i-1}, x \neq h_{i}\right\} .
$$

If $\omega_{i}$ is the restriction of $\omega$ to $M\left(X_{i}\right)$ then $H_{i}$ is, relative to $X_{i}$ and $\omega_{i}$, a weighted Hall set of finite type which has the dominance property. Let $\gamma_{i}$ be the dominance function for $H_{i}$ and let $\delta_{i}=\operatorname{ad}\left(h_{i}\right)$. Then, for all $u \in H_{i}$, $\delta_{i}(u)=\gamma_{i}(u)+w$ where $w$ is a linear comination of elements of $H_{i}$ which are strictly less than $\gamma_{i}(u)$.

Since $r \in L\left(X_{1}\right)$, we have $s \in H_{1}$ and we have the canonical decompositions in $H_{1}$

$$
\begin{gathered}
s=\operatorname{ad}\left(u_{1}\right) \operatorname{ad}\left(u_{2}\right) \cdots \operatorname{ad}\left(u_{m}\right)(x), \\
s_{i}=\gamma_{1}^{i}(s)=\operatorname{ad}\left(u_{1}\right) \operatorname{ad}\left(u_{2}\right) \cdots \operatorname{ad}\left(u_{m}\right)\left(\delta_{1}^{i}(x)\right),
\end{gathered}
$$

and $\delta_{1}^{i}(r)=s_{i}+w_{i}$ where $w_{i}$ is a linear combination of elements of $H_{1}$ which are strictly less than $s_{i}$. If $\mathfrak{r}$ is the ideal of $L$ generated by $r$ then $\mathfrak{r} \subset L\left(X_{1}\right)$ and $\mathfrak{r}$ is the ideal of $L\left(X_{1}\right)$ generated by the elements $r_{i}=\delta_{1}^{i}(r)$.

If $m=0$, i.e. if $\ell_{1}(s)=1$, then the images of the elements $r_{i}$ in the free $K$-module $L\left(X_{2}\right) /\left[L\left(X_{2}\right), L\left(X_{2}\right)\right]$ are part of a basis and so the $r_{i}$ are part of a basis of $L\left(X_{1}\right)$ (cf. [4, Proposition 2]). It follows that $\mathfrak{h}=L\left(X_{1}\right) / \mathfrak{r}$ is a free Lie algebra over $K$ and $\mathfrak{g} / \mathfrak{h} \cong K h_{1}$ with $\omega\left(h_{1}\right)<d$ since $r$ is not linear. Under our hypotheses on $r, x>h_{3}$, so the rank of $h$ is $\geq 2$.

Suppose that $m>0$ and let $h \in H_{1}$ be the smallest element of $X_{1}$ that appears in any of the elements $\delta_{1}^{i}(r)$. Then $h_{2} \leq h \leq u_{m}$. If $h>h_{2}$ then all the elements $r_{i}$ are in $L\left(X_{2}\right)$, the elements $u_{i}$ are in $H_{2}, x \in X_{2}$, and $r_{i j}=\delta_{2}^{j}\left(r_{i}\right)=s_{i j}+w_{i j}$ where $s_{i j}=\gamma_{2}^{j}\left(s_{i}\right) \in H_{2}$ with canonical decomposition in $\mathrm{H}_{2}$

$$
s_{i j}=\operatorname{ad}\left(u_{1}\right) \operatorname{ad}\left(u_{2}\right) \cdots \operatorname{ad}\left(u_{m}\right)\left(\delta_{2}^{j} \delta_{1}^{i}(x)\right)
$$

and $w_{i j}$ is a linear combination of elements of $H_{2}$ which are strictly smaller than $s_{i j}$. The same thing happens if $h=h_{2}<u_{m}$. Indeed, in this case, $u_{1}, \ldots, u_{m} \in L_{2}$ and $w_{i j} \in H_{2}$ since a nonzero scalar multiple of $h_{2}$ cannot appear as a term in the decomposition of $w_{i j}$ as a linear combination of elements of $H_{1}$ because $\omega\left(h_{2}\right)<\omega\left(w_{i j}\right)=\omega\left(s_{i j}\right)$ since $\ell_{2}\left(s_{i j}\right)>1$. If 
$h=h_{2}=u_{m}=\cdots=u_{m-k+1}<u_{m-k}$ then $s, s_{i j} \in H_{2}$ with canonical decompostions in $\mathrm{H}_{2}$

$$
\begin{gathered}
s=\operatorname{ad}\left(u_{1}\right) \operatorname{ad}\left(u_{2}\right) \cdots \operatorname{ad}\left(u_{m-k}\right)\left(\delta_{2}^{k}(x)\right), \\
s_{i j}=\operatorname{ad}\left(u_{1}\right) \operatorname{ad}\left(u_{2}\right) \cdots \operatorname{ad}\left(u_{m-k}\right)\left(\delta_{2}^{j+k} \delta_{1}^{i}(x)\right),
\end{gathered}
$$

and, as above, $w_{i j}$ is a linear combination of elements of $H_{2}$ if $m \neq k$. If $m=k$, we have

$$
\omega\left(w_{i j}\right)=\omega\left(s_{i j}\right)>\omega\left(h_{2}\right)
$$

and so $w_{i j} \in H_{2}$.

Proceeding inductively, assume that $r \in L\left(X_{n}\right)$ and that for $i=\left(i_{1}, \ldots, i_{n}\right) \in$ $\mathbb{N}^{n}, n \geq 1$,

$$
r_{i}=\delta_{n}^{i_{n}} \cdots \delta_{2}^{i_{2}} \delta_{1}^{i_{1}}(r)=s_{i}+w_{i},
$$

where $s_{i} \in H_{n}$ with canonical decomposition in $H_{n}$

$$
s_{i}=\operatorname{ad}\left(u_{1}\right) \operatorname{ad}\left(u_{2}\right) \cdots \operatorname{ad}\left(u_{m-k}\right)\left(y_{i}\right)
$$

with

$$
y_{i}=\delta_{n}^{i_{n}+k_{n}} \cdots \delta_{2}^{i_{2}+k_{2}} \delta_{1}^{i_{1}}(x) \quad\left(k_{i} \geq 0, \text { uniquely determined by } s\right),
$$

and $w_{i}$ a linear combination of elements of $H_{n}$ which are strictly less than $s_{i}$.

If $k=m$ the elements $s_{i} \in X_{n}$ are distinct and so the images of the elements $r_{i}$ in the free $K$-module $L\left(X_{n}\right) /\left[L\left(X_{n}\right), L\left(X_{n}\right)\right]$ are part of a basis. Hence the $r_{i}$ are part of a basis of the free Lie algebra $L\left(X_{n}\right)$. Since they also generate $\mathfrak{r}$ as an ideal of $L\left(X_{n}\right)$ we obtain that $\mathfrak{h}=L\left(X_{n}\right) / \mathfrak{r}$ is a free Lie algebra with

$$
\mathfrak{g} / \mathfrak{h} \cong K h_{1} \oplus \cdots \oplus K h_{n} .
$$

Since $h_{n}=u_{1}$, we have $\omega\left(h_{n}\right)<d$ and so the components of $\mathfrak{g} / \mathfrak{h}$ are zero in degrees $\geq d$. The rank of $\mathfrak{h}$ is infinite since, in this case, $s_{i} \notin X_{1}$ and so the the $r_{i}$ together with the elements $\delta_{1}^{j}\left(h_{2}\right)>h_{n}$ are part of a basis of $L\left(X_{n}\right)$.

Remark. In the case $m=k$ it is possible to continue the elimination if $r \in$ $L\left(X_{n+1}\right)$. This happens iff either $r$ is not a linear form in $L\left(X_{n}\right)$ or $r$ is a linear form in $L\left(X_{n}\right)$ which does not have a scalar multiple of $h_{n+1}$ as a term. In this case, we get

$$
r_{i, i_{n+1}}=\delta_{n+1}^{i_{n+1}}\left(r_{i}\right)=s_{i, i_{n+1}}+w_{i, i_{n+1}}
$$

with $s_{i, i_{n+1}} \in X_{n+1}$,

$$
s_{i, i_{n+1}}=\delta_{n+1}^{i_{n+1}} \delta_{n}^{i_{n}+k_{n}} \cdots \delta_{2}^{i_{2}+k_{2}} \delta^{i_{1}}(x),
$$

and $w_{i, i_{n+1}}$ a linear combination of elements of $H_{n+1}$ which are stricly less than $s_{i, i_{n+1}}$. We make use of the fact that $L\left(X_{n+1}\right)$ is an ideal of $L(X)$.

If $k<m$ let $h$ be the smallest element of $X_{n}$ that appears in any of the $r_{i}$. Then $h_{n+1} \leq h \leq u_{m-k}$. If $h>h_{n+1}$ then $u_{i}, w_{i} \in H_{n+1}, y_{i} \in X_{n+1}$ and

$$
\delta_{n+1}^{i_{n+1}}\left(r_{i}\right)=s_{i, i_{n+1}}+w_{i, i_{n+1}}
$$

with $s_{i, i_{n+1}} \in H_{n}$ with canonical decomposition in $H_{n+1}$

$$
s_{i, i_{n+1}}=\operatorname{ad}\left(u_{1}\right) \operatorname{ad}\left(u_{2}\right) \cdots \operatorname{ad}\left(u_{m-k}\right) \delta_{n+1}^{i_{n+1}}\left(y_{i}\right)
$$

and $w_{i, i_{n+1}}$ a linear combination of elements of $H_{n+1}$ which are strictly less than $s_{i, i_{n+1}}$. The same is true if $h=h_{n+1}<u_{m-k}$. Indeed, a nonzero scalar 
multiple of $h_{n+1}$ cannot appear as a term in the decomposition of $w_{i}$ as a linear combination of elements of $H_{n}$; otherwise, $\omega\left(h_{n+1}\right)=\omega\left(s_{i}\right)$ contradicting $\ell_{n}\left(s_{i}\right) \geq 2$. If $h=h_{n+1}=u_{m-k}=\cdots u_{m-k-\ell+1}<u_{m-k-\ell}$ then again $s_{i, i_{n+1}} \in$ $H_{n+1}$ with canonical decompostion in $H_{n+1}$

$$
s_{i, i_{n+1}}=\operatorname{ad}\left(u_{1}\right) \operatorname{ad}\left(u_{2}\right) \cdots \operatorname{ad}\left(u_{m-k-\ell}\right) \delta_{n+1}^{i_{n+1}+\ell}\left(y_{i}\right)
$$

and $w_{i, i_{n+1}} \in L\left(X_{n+1}\right)$ since

$$
\omega\left(w_{i, i_{n+1}}\right)=\omega\left(s_{i, i_{n+1}}\right)>\omega\left(h_{n+1}\right) .
$$

If $n$ is smallest with $s \in X_{n}$, it follows that the above procedure gives a free subalgebra $\mathfrak{h}$ of $\mathfrak{g}$ in $n$ steps. Since $L\left(X_{n}\right)$ is an ideal of $L$, we obtain that $\mathfrak{h}$ is an ideal of $\mathfrak{g}$. The free subalgebra $\mathfrak{h}$ is of infinite rank if $n>1$ or $n=1$ and $\operatorname{Card}(X)>2$. If $n=1$ we have $\mathfrak{h}$ of finite rank $\geq 2$ if $\operatorname{Card}(X)=2$. Finally, we may take $g_{i}$ to be the image of $h_{i}$ in $g$ since

$$
\mathfrak{g} / \mathfrak{h} \cong K h_{1} \oplus K h_{2} \oplus \cdots \oplus K h_{n}
$$

and $\omega\left(h_{i}\right)<d$ for $1 \leq i \leq n$.

Remarks. The natural number $n$ and the Lie element $s$ could have been chosen more optimally as follows: Let $n$ be smallest with $r \in L\left(X_{n}\right), r \notin$ $\left[L\left(X_{n}\right), L\left(X_{n}\right)\right]$ and such that the largest linear term of $r$ in $L\left(X_{n}\right)$ is a unit multiple of $s \in X_{n}$. The terms of $r$ which are in $\left[L\left(X_{n}\right), L\left(X_{n}\right)\right]$ cause no problems since $L\left(X_{n}\right)$ is an ideal of $L(X)$ and so they can be ignored. Also note that, if $n$ is largest with $r \in L\left(X_{n}\right)$, then $r$ is a linear form in $L\left(X_{n}\right)$.

We now consider the general case. Let $n$ be largest with $r \in L\left(X_{n}\right)$. Then $r$ is a homogeneous linear form in $L\left(X_{n}\right)$ whose image in $L\left(X_{n}\right) \otimes(K / \mathfrak{m})$ is nonzero for any maximal ideal of $K$. It follows that the homogeneous components of $\mathfrak{h}$ and $\mathfrak{h} /[\mathfrak{h}, \mathfrak{h}]$ are locally free of constant finite rank and hence that $\mathfrak{h}$ and $\mathfrak{h} /[\mathfrak{h}, \mathfrak{h}]$ are free $K$-modules. Since $\mathfrak{h} \otimes(K / \mathfrak{m})$ is a free Lie algebra over $K / \mathfrak{m}$ for each maximal ideal $\mathfrak{m}$ of $K$ by [9, Satz 5] or [4, Proposition 2], it follows that $\mathfrak{h}$ is free over $K$ by [4, Proposition 2] and the following Lemma:

Lemma. Let $\mathfrak{g}$ be a graded Lie algebra over a principal ideal domain $K$ such that each homogeneous component is a finitely generated free $K$-module and such that $\mathfrak{g} /[\mathfrak{g}, \mathfrak{g}]$ is also $K$ free. If the homology group $H_{2}(\mathfrak{g}, K / \mathfrak{m})=0$ for each maximal ideal $\mathfrak{m}$ of $K$ then $H_{2}(\mathfrak{g}, K)=0$.

Proof. We choose a presentation $\mathfrak{g}=L / \mathfrak{r}$ together with a grading of the free Lie algebra $L$ such that $L /[L, L]$ is isomorphic to $\mathfrak{g} /[\mathfrak{g}, \mathfrak{g}]$ as graded modules. Using the Hochschild-Serre spectral sequence for the extension $\mathfrak{g}=L / \mathfrak{r}$ :

$$
H_{2}(L, K) \rightarrow H_{2}(\mathfrak{g}, K) \rightarrow H_{0}\left(\mathfrak{g}, H_{1}(\mathfrak{r}, K)\right) \rightarrow H_{1}(L, K) \rightarrow H_{1}(\mathfrak{g}, K) \rightarrow 0,
$$

cf. [3, p. 351], and the fact that $H_{2}(L, K)=0, H_{1}(\mathfrak{g}, K)=\mathfrak{g} /[\mathfrak{g}, \mathfrak{g}]$ and

$$
H_{0}\left(\mathfrak{g}, H_{1}(\mathfrak{r}, K)\right)=\mathfrak{r} /[\mathfrak{r}, L],
$$

we obtain that $H_{2}(\mathfrak{g}, K)$ is isomorphic to $\mathfrak{r} /[\mathfrak{r}, L]$. Since $H_{2}(\mathfrak{g}, K / \mathfrak{m})$ is isomorphic to $(L /[\mathfrak{r}, L]) \otimes(K / \mathfrak{m})$ we obtain that $(\mathfrak{r} /[\mathfrak{r}, L]) \otimes(K / \mathfrak{m})=0$ for all maximal ideals $\mathfrak{m}$ of $K$ and hence that $\mathfrak{r} /[\mathfrak{r}, L]=0$ since each homogeneous component of $\mathfrak{r} /[\mathfrak{r}, L]$ is a finitely generated $K$-module.

The following is a corollary of the proof. 
Corollary. Let $K$ be an arbitrary commutative ring and let $r$ be a homogeneous element of $L(X)$. Suppose that for some $n$ we have $r \in L\left(X_{n}\right), r \notin$ $\left[L\left(X_{n}\right), L\left(X_{n}\right)\right]$ and that the largest linear term of $r$ in $L\left(X_{n}\right)$ has a coefficient which is a unit of $K$. Then the elements

$$
\operatorname{ad}\left(h_{n}\right)^{i_{n}} \ldots \operatorname{ad}\left(h_{2}\right)^{i_{2}} \operatorname{ad}\left(h_{1}\right)^{i_{1}}(r) \quad\left(i_{1}, \ldots i_{n} \geq 0\right)
$$

are part of a basis of $L\left(X_{n}\right)$. In particular, Theorem 1 holds in this case.

\section{ProOF OF THEOREM 2}

We may assume that $X=\left\{x_{1}, \ldots, x_{n}\right\}$. Let

$$
r=r_{1}+r_{2}+\cdots+r_{d}
$$

be the decomposition of $r$ into its homogeneous components $r_{i}$ of degree $i$ for the natural grading of $L$.

Lemma. If $K$ is a field and the center of $\mathfrak{g}=L /(r)$ is nonzero then so is the center of $L /\left(r_{d}\right)$.

Proof. Let $\pi$ be an indeterminate over $K$ and let $L^{\prime}$ (resp. $L^{\prime \prime}$ ) be $L \otimes_{K} K[\pi]$ (resp. $L \otimes_{K} K(\pi)$ ). Similarly define $\mathfrak{g}^{\prime}, \mathfrak{g}^{\prime \prime}$. If $\psi$ is the automorphism of $L^{\prime \prime}$ defined by $\psi\left(x_{i}\right)=x_{i} / \pi$, let $s=\pi^{d} \psi(r)$. Then

$$
s=r_{d}+\pi r_{d-1}+\cdots+\pi^{d-1} r_{1}
$$

with $s, t \in L^{\prime}$ and

$$
\mathfrak{g}^{\prime \prime} \cong\left(L^{\prime} /(s)\right) \otimes_{K[\pi]} K(\pi) .
$$

By $\left[4\right.$, Theorem 3], $L^{\prime} /(s)$ is a free $k[\pi]$ module. Now suppose that the center of $\mathfrak{g}$ is nonzero. Then the center of $\mathfrak{g}_{\pi}=L^{\prime} /(s)$ is nonzero and hence so is the center of $\mathfrak{g}_{\pi} / \pi \mathfrak{g}_{\pi} \cong L /\left(r_{d}\right)$.

Suppose $K$ is a field and that the center of $\mathfrak{g}$ is not trivial. Then, by the above Lemma, the center of $L /\left(r_{d}\right)$ is not zero. If $d=1$ we must have $\operatorname{Card}(X)$ $=2$. Suppose that either $\operatorname{Card}(X)>2$ or $\operatorname{Card}(X)=2, d>2$ and let $z$ be a nonzero homogeneous element of the center of $L /\left(r_{d}\right)$. We first show that the degree of this element must be $\geq d$. Let $x_{1}, x_{2}$ be the first two elements of $X$. Since $\left[x_{1}, z\right] \in \mathfrak{r}$ the degree of $z$ is $\geq d-1$. If the degree of $z$ is $d-1$ then $\left[x_{i}, z\right]=a_{i} r$ with $a_{i} \in K, a_{i} \neq 0$. Hence $\left[a_{2} x_{1}, z\right]=\left[a_{1} x_{2}, z\right]$ which gives $\left[z, a_{2} x_{1}-a_{1} x_{2}\right]=0$. This implies that $z=a x_{1}+b x_{2}$ and $r=c\left[x_{1}, x_{2}\right]$. But then, by hypothesis, we must have $\operatorname{Card}(X)>2$. If $x_{3}$ is the third element of $X_{3}$ the above argument yields $r=d\left[x_{1}, x_{3}\right]$ which is a contradiction. Hence the degree of $z$ is at least $d$ which implies, by Theorem 1 , that $z \in \mathfrak{h}$ and hence that $z=0$ since the rank of $\mathfrak{h}$ is at least 2 . This contradiction means that we must have $\operatorname{Card}(X)=2$ and $d=1$ or $d=2$. If $d=1$ we are done so we may assume $d=2$. If $r_{1}=0$ we are again done, so we may assume $r=a x_{1}+b x_{2}+c\left[x_{1}, x_{2}\right]$ with $c \neq 0$ and one of $a, b$ nonzero. After a linear change of variables we have $r=x_{1}+\left[x_{1}, x_{2}\right]$. But, in this case, the center of $\mathfrak{g}$ is zero; so this case does not arise.

We now consider the case where $K$ is a principal ideal domain and $r$ is homogeneous. Without loss of generality, we may assume that $d>2$ or $\operatorname{Card}(X)$ $>2$. Suppose first that $r$ is not a proper multiple of another element of $L$. 
Then, by [4, Corollary to Theorem 2], $\mathfrak{g}=L /(r)$ is a free $K$-module. If $K^{\prime}$ is the quotient field of $K$, the center of $\mathfrak{g}$ is zero iff the center of $\mathfrak{g} \otimes_{K} K^{\prime}$ is zero which gives Theorem 2 .

If $r$ is a proper multiple of another element of $L$, we can write $r=c s$ with $c$ a nonunit of $K$ and $s$ an element of $L$ which is not a proper multiple. Let $\mathfrak{s}=(s)$. Then $(r)=c \mathfrak{s}$ and $\mathfrak{g}=L / c \mathfrak{s}$. Since $L / \mathfrak{s}$ is a free $K$-module by [4, Corollary to Theorem 2], we obtain that $s$ is a free Lie-algebra over $K$, cf. [9, Satz 5]. Hence $s / c s$ is a free Lie algebra over $K / K c$. Since this Lie algebra is of infinite rank, its center is zero, and since the center of $L / \mathfrak{s}$ is zero by the first part we see that the center of $\mathfrak{g}$ must also be zero.

\section{APPLICATION TO PRO- $p$-GROUPS}

Let $X$ be a well-ordered set together with a locally finite weight function $\omega$ which is compatible with the ordering of $X$. We may therefore assume $X=\left\{x_{1}, x_{2}, \ldots.\right\}$ with $x_{i}<x_{j}$ if $i<j$. Let $F=F(X)$ be the free pro- $p$ group on the set $X$, cf. $[7, \S 1.5]$. Let $H$ be a weighted Hall set with respect to $X, \omega$ having the dominance property and let the sets $X_{n} \subset M(X)(n \geq 0)$ be defined as in the proof of Theorem 1. Using the embedding of $M(X)$ into $F$ obtained by means of the operation $[x, y]=x^{-1} y^{-1} x y=x^{-1} y^{x}$ on $F$, we identify $X_{n}$ with its image in $F$. We also define $\operatorname{ad}(x)(y)=[x, y]$. The following result is the elimination theorem for pro-p-groups:

Proposition 7. Let $f: F(X) \rightarrow F(T)$ be the homomorhism of pro-p-groups defined by $f(x)=x$ for $x \in T$ and $f(x)=1$ for $x \in X-T$. Then $\operatorname{ker}(f)$ is a free pro-p-group with basis the set $X_{T}$ consisting of elements

$$
x_{i, j}=\operatorname{ad}\left(x_{j_{1}}\right) \operatorname{ad}\left(x_{j_{2}}\right) \cdots \operatorname{ad}\left(x_{j_{k}}\right)\left(x_{i}\right)
$$

with $x_{j_{1}}, x_{j_{2}}, \ldots, x_{j_{k}} \in T(k \geq 0)$ and $x_{i} \in X-T$.

Proof. For the natural right action of $F$ on $N=\operatorname{ker}(f)$ we have

$$
x_{i j}^{x_{\ell}}=x_{i j} x_{i, j^{\prime}}^{-1}
$$

with $j^{\prime}=\left(\ell, x_{j_{1}}, \ldots, x_{j_{k}}\right)$ if $j=\left(x_{j_{1}}, \ldots, x_{j_{k}}\right)$. This also defines a right action of $F(T)$ on $\left.F\left(X_{T}\right)\right)$. Let $h_{0}$ be the homomorphism of $F\left(X_{T}\right)$ into $N$ induced by the identity map on $X_{T}$ and let $S$ be the semidirect product of $F\left(X_{T}\right)$ by $F(T)$. Since $h_{0}$ is compatible with the actions of $F(T)$ on $F\left(X_{T}\right)$ and $N$ it extends to a map $h$ of $S$ into $F(X)$. If $g$ is the homomorphism of $F(X)$ into $S$ with $g(x)=(1, x)$ for $x \in X-T$ and $g(x)=(x, 1)$ for $x \in T$ then $h g=1$ and $g h=1$.

Corollary. If $f$ is the homomorphism of $F(X)$ into $F\left(x_{1}\right)$ defined by $f\left(x_{1}\right)=$ $x_{1}$ and $f(x)=1$ for $x \in X, x \neq x_{1}$, then $\operatorname{ker}(f)=F\left(X_{1}\right)$.

If $F_{n}$ is the closed subgroup of $F$ generated by $X_{n}$ then $F_{n}$ is a normal subgroup of $F$ and is a free pro-p-group with basis $X_{n}$. The weight function $\omega$ defines a filtration of $F$ and induces one on $F_{n}$ (cf. [5]) so that, if $\operatorname{gr}\left(F_{n}\right)$ is the associated Lie algebra, we have $\operatorname{gr}\left(F_{n}\right)=L\left(X_{n}\right)$ over $\mathbb{Z}_{p}$.

Theorem 5. Let $r \in F$ and suppose that $r \in F_{n}, r \notin F_{n}^{p}\left[F_{n}, F_{n}\right]$. Let $G_{i}$ be the image of $F_{i}$ in $G$. Then $G_{n}$ is a normal free pro-p-subgroup of $G$ 
and $\Gamma_{i}=G_{i-1} / G_{i} \cong \mathbb{Z}_{p}$ for $0<i \leq n$. Moreover, $R /[R, R]$ is a free $\mathbb{Z}_{p}[[G]]-$ module of rank 1 , where $\mathbb{Z}_{p}[[G]]$ is the completed $\mathbb{Z}_{p}$-algebra of $G$. In particular, $G$ is of cohomological dimension $\leq 2$.

Proof. For $i>0$ let $g_{i}$ be the image of $h_{i}$ in $\Gamma_{i-1}$, where $h_{i}$ is the smallest element of $X_{i-1}$. Then $g_{i}$ is a generator of $\Gamma_{i}$. Let $\gamma_{i}=1-g_{i}$ in $\mathbb{Z}_{p}[[G]]$. For $i=\left(i_{1}, \ldots, i_{n}\right) \in \mathbb{N}^{n}$ let

$$
r_{i}=\operatorname{ad}\left(h_{n}\right)^{i_{n}} \cdots \operatorname{ad}\left(h_{2}\right)^{i_{2}} \operatorname{ad}\left(h_{1}\right)^{i_{1}}(r)
$$

and let $\rho$ (resp. $\rho_{i}$ ) be the image of $r$ (resp. $r_{i}$ ) in $R /[R, R]$. The elements $r_{i}$ generate $R$ as a closed normal subgroup of $F_{n}$ and

$$
\rho_{i}=\rho \cdot\left(\gamma_{1}^{i_{1}} \gamma_{2}^{i_{2}} \cdots \gamma_{n}^{i_{n}}\right)
$$

for the natural right action of $\mathbb{Z}_{p}[[G]]$ on $R /[R, R]$. We now show that the images of the $\rho_{i}$ in the free commutative pro-p-group $F_{n} /\left[F_{n}, F_{n}\right]$ are part of a basis. To see this let $\overline{\rho_{i}}$ (resp. $\bar{\rho}$ ) be the initial forms of the images of $\rho_{i}$ (resp. $\rho$ ) in $F_{n} / F_{n}^{p}\left[F_{n}, F_{n}\right]$, with respect to the filtration defined by $\omega$. It suffices to show that the $\overline{\rho_{i}}$ can be completed to a homogeneous basis of $\operatorname{gr}\left(F_{n} / F_{n}^{p}\left[F_{n}, F_{n}\right]\right.$. But this follows from the Corollary to Theorem 1 with $K=\mathbb{F}_{p}$ and $r=\bar{\rho} ;$ note that in $L(X) \otimes_{\mathbb{Z}_{p}} \mathbb{Z} / p \mathbb{Z}$

$$
\overline{\rho_{i}}=\operatorname{ad}\left(h_{n}\right)^{i_{n}} \cdots \operatorname{ad}\left(h_{1}\right)^{i_{1}}(\bar{\rho}) .
$$

If $S$ is the set of the elements $r_{i}$, we obtain that $S$ is part of a basis $Y$ of the free pro-p-group $F_{n}$. This yields $G_{n}=F(T)$ with $T=Y-S$. If $I=\mathbb{N}^{n}$ then, by the elimination theorem, the mapping which sends the element $u=\left(u_{i}\right)_{i \in I} \in Z_{p}\left[\left[G_{n}\right]\right]^{I}$ to the element $\sum \rho_{i} \cdot u_{i} \in R /[R, R]$ is an isomorphism of $\mathbb{Z}_{p}\left[\left[G_{n}\right]\right]$-modules. But every element of $\mathbb{Z}_{p}[[G]]$ can be uniquely written in the form

$$
\sum \gamma_{1}^{i_{1}} \gamma_{2}^{i_{2}} \cdots \gamma_{n}^{i_{n}} u_{i_{1} i_{2} \ldots i_{n}}
$$

with $u_{i_{1} i_{2} \ldots i_{n}} \in \mathbb{Z}_{p}\left[\left[G_{n}\right]\right]$. This implies that $R /[R, R]$ is a free $\mathbb{Z}_{p}[[G]]$-module. By [4, Proposition 1], we obtain that $G$ is of cohomological dimension $\leq 2$.

As an example, $\operatorname{cd}(G)=2$ if $r$ is the relator

$$
\left[x_{1},\left[x_{1}, x_{2}\right]\right]^{p} \operatorname{ad}\left(\left[x_{1}, x_{2}\right]\right)^{m}\left(\left[x_{1},\left[x_{1}, x_{2}\right]\right]\right) \text {. }
$$

This follows from the fact the hypothesis of Theorem 5 is satisfied with $n=3$. The best one could do for this relator, using the results of [4], was to prove that $\operatorname{cd}(G)=2$ for $p>\frac{2}{3} m+1$.

\section{REFERENCES}

1. N. Bourbaki, Groupes et algèbres de Lie, Chapitre 2, Hermann, Paris, 1972.

2. H. Bass and A. Lubotsky, Linear-central filtrations on groups, The Mathematical Legacy of Wilhelm Magnus: Groups, Geometry and Special Functions (W. Abikoff, J.S. Birman and K. Kuiken, eds.), Contemporary Math., vol. 169, Amer. Math. Soc., Providence, RI, 1994, pp. 45-98.

3. H. Cartan and S. Eilenberg, Homological algebra, Princeton University Press, Princeton, NJ, 1956.

4. J.P. Labute, Algèbres de Lie et pro-p-groupes définis par une seule relation, Invent. Math 4 (1967), 142-158. 
5. M. Lazard, Groupes analytiques p-adiques, I.H.E.S. Publ. Math. 26 (1965), 389-603.

6. K. Murasugi, The center of a group with a single defining relation, Math. Ann. 155 (1964), 246-251.

7. J-P. Serre, Cohomologie Galoisienne, Springer-Verlag, Berlin-Heidelberg-New York, 1965.

8. A. I. Širšov, Some algorithmic problems for Lie algebras, Sibirsk. Math. Ž. 3 (1962), 292-296. (Russian)

9. E. Witt, Die Unterringe der freien Lieschen Ringe, Math. Z. 64 (1956), 195-216.

805 Sherbrooke Street West, Montreal, Quebec H3A 2K6, Canada

E-mail address: labute@math.mcgill.ca 